Supplement of Biogeosciences, 13, 5849-5863, 2016

http://www.biogeosciences.net/13/5849/2016/

doi:10.5194/bg-13-5849-2016-supplement

(C) Author(s) 2016. CC Attribution 3.0 License.

(c) (i)

Supplement of

\title{
The importance of freshwater systems to the net atmospheric exchange of carbon dioxide and methane with a rapidly changing high Arctic watershed
}

Craig A. Emmerton et al.

Correspondence to: Craig A. Emmerton (emmerton@ualberta.ca)

The copyright of individual parts of the supplement might differ from the CC-BY 3.0 licence. 


\section{Bottle - Automated system dissolved $\mathrm{CO}_{2}$ concentration comparison}

Though bottle (time-series) and automated system (diurnal) dissolved $\mathrm{CO}_{2}$ concentrations were not directly compared in this study, the concentrations measured in Pond 01 and Skeleton Lake using each approach were near identical in most cases (Figure S7). In 2008 (Skeleton Lake) and 2009 (Pond 01), however, calibration of the automated systems appeared to have shifted during transit, causing a slight step difference in measured $\mathrm{CO}_{2}$ concentrations between the two approaches.

\section{Ebullition fluxes}

Ebullition can also liberate $\mathrm{CO}_{2}$ and $\mathrm{CH}_{4}$ from freshwater systems. We used submerged inverted 30-cm plastic funnels with a bubble collection chamber to quantify ebullition fluxes of $\mathrm{CO}_{2}$ and $\mathrm{CH}_{4}$ from the surface of Skeleton Lake and Pond 01. Traps were deployed continuously at both sites during the 2007 and 2008 summers and checked weekly for bubble volume accumulation. Ebullition volume was measured by drawing into a syringe, through a rubber septum in the collection chamber, the accumulated gas. However, we did not measure gas concentrations of this trapped gas because $\mathrm{CO}_{2}$ and $\mathrm{CH}_{4}$ can diffuse back into surface waters while sitting in the trap. Instead, fresh bubbles were collected for $\mathrm{CO}_{2}$ and $\mathrm{CH}_{4}$ analyses by probing the sediments and collecting them into a hand held bubble trap. Samples were then immediately transferred to evacuated, stoppered $30 \mathrm{ml}$ Wheaton bottles and analyzed for $\mathrm{CO}_{2}$ and $\mathrm{CH}_{4}$ concentrations on the $\mathrm{GC}$ in a manner similar to that described in the main manuscript for water samples. Bubble $\mathrm{CO}_{2}$ and $\mathrm{CH}_{4}$ concentrations were multiplied by bubble volume collected over the weeklong period to determine ebullition fluxes. 


\section{Tables}

Table S1: Mean weather conditions measured by a polar semidesert eddy covariance/meteorological station about $1 \mathrm{~km}$ from base camp during the growing season between 2008 and 2012 in the Lake Hazen watershed.

\begin{tabular}{|c|c|c|c|c|c|c|c|}
\hline Month & $\begin{array}{r}\text { Air } \\
\text { temp. } \\
\left({ }^{\circ} \mathbf{C}\right)\end{array}$ & $\begin{array}{r}\text { Rainfall } \\
(\mathbf{m m})\end{array}$ & $\begin{array}{l}\text { Wind } \\
\text { speed } \\
\text { (kph) }\end{array}$ & $\begin{array}{r}\text { PAR } \\
\left(\mu \mathrm{mol} \mathrm{m}{ }^{-2} \mathrm{~s}^{-1}\right)\end{array}$ & $\begin{array}{r}\text { Air } \\
\text { pressure } \\
(\mathrm{kPa})\end{array}$ & $\begin{array}{r}\text { Soil } \\
\text { moisture } \\
\left(\mathbf{m}^{3} \mathbf{m}^{-3}\right)\end{array}$ & $\begin{array}{r}\text { Soil } \\
\text { temp. } \\
\left({ }^{\circ} \mathrm{C}\right)\end{array}$ \\
\hline & 5.0 & & 2.9 & 699 & 99.4 & 0.16 & 6.9 \\
\hline & 8.4 & 19.3 & 2.9 & 596 & 98.8 & 0.15 & 12.0 \\
\hline August & 5.7 & 10.5 & 2.7 & 384 & 98.7 & 0.14 & 7.4 \\
\hline
\end{tabular}

Table S2 Sampling years and dates for greenhouse gases concentrations collected using bottles (B) or automated systems (AS), and general chemical analyses (C) of several freshwater systems throughout the Lake Hazen watershed.

\begin{tabular}{lrrrrrrrr}
\hline Water body & & $\mathbf{2 0 0 5}$ & $\mathbf{2 0 0 7}$ & $\mathbf{2 0 0 8}$ & $\mathbf{2 0 0 9}$ & $\mathbf{2 0 1 0}$ & $\mathbf{2 0 1 1}$ & $\mathbf{2 0 1 2}$ \\
\hline \multirow{2}{*}{ Pond 01 } & $\mathrm{B}$ & $6-21 / 7$ & $24 / 6-21 / 7$ & $6 / 7-4 / 8$ & $29 / 6-22 / 7$ & $16 / 6-20 / 7$ & $6 / 7-30 / 7$ & - \\
& $\mathrm{AS}$ & - & $24 / 6-21 / 7$ & $10 / 7-2 / 8$ & $29 / 6-21 / 7$ & $19 / 6-5 / 7$ & - & - \\
& $\mathrm{C}$ & $6-23 / 7$ & $28 / 6-18 / 7$ & $9 / 7-2 / 8$ & $2-22 / 7$ & $28 / 6-20 / 7$ & & - \\
\hline \multirow{2}{*}{ Pond 02 } & $\mathrm{B}$ & $6-21 / 7$ & $8 / 7$ & $9 / 7-2 / 8$ & - & $10-20 / 7$ & $6 / 7-30 / 7$ & - \\
& $\mathrm{C}$ & $6-22 / 7$ & $6 / 7$ & $9 / 7-2 / 8$ & - & $10-20 / 7$ & - & - \\
\hline \multirow{2}{*}{ Pond 03 } & $\mathrm{B}$ & $10 / 7$ & $13-14 / 7$ & $29 / 7$ & - & $12-17 / 7$ & $6 / 7-30 / 7$ & - \\
& $\mathrm{C}$ & - & $14 / 7$ & - & - & $12-17 / 7$ & - & - \\
\hline \multirow{2}{*}{ Pond 07 } & $\mathrm{B}$ & $15 / 7$ & $9 / 7$ & $29 / 7$ & - & $13-18 / 7$ & $6 / 7-30 / 7$ & - \\
& $\mathrm{C}$ & - & $10 / 7$ & - & - & $13-18 / 7$ & - & - \\
\hline \multirow{2}{*}{ Pond 10 } & $\mathrm{B}$ & $15 / 7$ & - & - & - & $13-18 / 7$ & $6 / 7-30 / 7$ & - \\
& $\mathrm{C}$ & - & - & - & - & $13-18 / 7$ & - & - \\
\hline \multirow{2}{*}{ Pond 11 } & $\mathrm{B}$ & $15 / 7$ & - & - & - & $12-17 / 7$ & $4 / 7-30 / 7$ & $4 / 7-31 / 7$ \\
& $\mathrm{C}$ & - & - & - & - & $12-17 / 7$ & - & $31 / 7$ \\
\hline \multirow{2}{*}{ Pond 12 } & $\mathrm{B}$ & $15 / 7$ & $14-16 / 7$ & $29 / 7$ & - & $12-17 / 7$ & - & - \\
& $\mathrm{C}$ & - & $14 / 7$ & - & - & $12-17 / 7$ & - & - \\
\hline \multirow{2}{*}{ Pond 16 } & $\mathrm{B}$ & - & - & - & - & $13-18 / 7$ & $6 / 7-30 / 7$ & \\
& $\mathrm{C}$ & - & - & - & - & $13-18 / 7$ & - & \\
\hline \multirow{2}{*}{ Skeleton Lake } & $\mathrm{BS}$ & - & $25 / 6-19 / 7$ & $6 / 7-3 / 8$ & $29 / 6-22 / 7$ & $18 / 6-19 / 7$ & $4 / 7-30 / 7$ & $4 / 7-31 / 7$ \\
& $\mathrm{C}$ & - & - & $8 / 7-3 / 8$ & $1-21 / 7$ & $25 / 6-20 / 7$ & - & - \\
\hline \multirow{2}{*}{ Lake Hazen } & $\mathrm{B}$ & $4-20 / 7$ & $24 / 6-21 / 7$ & $6 / 7-4 / 8$ & $29 / 6-22 / 7$ & $22 / 6-20 / 7$ & $6 / 7-30 / 7$ & - \\
Shoreline & $\mathrm{C}$ & $6-13 / 7$ & - & $10 / 7-3 / 8$ & $2-22 / 7$ & $28 / 6-20 / 7$ & - & - \\
\hline
\end{tabular}


Table S3 Empirical relationships for $\mathrm{k}\left(\mathrm{cm} \mathrm{hr}^{-1}\right.$; Hamilton et al., 1994) used in the mass flux equation for greenhouse gases samples (Equation 1 in the manuscript).

if $\mathrm{U}<3 \mathrm{~m} \mathrm{~s}^{-1}$ :

$$
\begin{array}{ll}
\text { if } \mathrm{U}<3 \mathrm{~m} \mathrm{~s}^{-1}: & \mathrm{k}_{600}=0.76 \mathrm{U} \\
& \mathrm{k}_{\mathrm{CO} 2}=\mathrm{k}_{600} *\left(600^{0.67} / \mathrm{SC}_{\mathrm{CO} 2}{ }^{0.67}\right) \\
& \mathrm{k}_{\mathrm{CH} 4}=\mathrm{k}_{600} *\left(600^{0.67} / \mathrm{SC}_{\mathrm{CO} 2}{ }^{0.67}\right) \\
& \mathrm{k}_{600}=5.6 \mathrm{U}-14.14 \\
& \mathrm{k}_{\mathrm{CO} 2}=\mathrm{k}_{600} *\left(600^{0.50} / \mathrm{SC}_{\mathrm{CO} 2}{ }^{0.50}\right) \\
& \mathrm{k}_{\mathrm{CH} 4}=\mathrm{k}_{600} *\left(60 \mathrm{~m} \mathrm{~s}^{-1}:\right.
\end{array}
$$

Notes: $U$ is in-situ wind speed $\left(\mathrm{m} \mathrm{s}^{-1}\right)$ measured at $1 \mathrm{~m}$ on automated systems or at a nearby meteorological station; $k_{600}\left(\mathrm{~cm} \mathrm{hr}^{-1}\right)$ is the exchange coefficient 
Table S4 Pearson correlation coefficients of greenhouse gases and general chemistry of freshwater types in the Lake Hazen watershed during the growing seasons of 2005, 2007-2012. Statistical significance at $\alpha=0.05$ indicated in bold (IBM SPSS Statistics 23).

\begin{tabular}{|c|c|c|c|c|c|c|c|c|}
\hline & \multicolumn{4}{|c|}{$\mathrm{CO}_{2}$} & \multicolumn{4}{|c|}{$\mathrm{CH}_{4}$} \\
\hline & Evap. & Melt. & Shore. & LH Shore & Evap. & Melt. & Shore. & LH Shore \\
\hline $\operatorname{Air}_{P}$ & .542 & .050 & .350 & $-.565^{*}$ & $-.803^{*}$ & .097 & .144 & -.429 \\
\hline Water $_{\mathrm{T}}$ & .131 & -.409 & .397 & -.308 & .258 & $-.483^{*}$ & .266 & .187 \\
\hline $\mathrm{CO}_{2}$ & 1 & 1 & 1 & 1 & -.526 & $.630^{* *}$ & $.530^{*}$ & .418 \\
\hline $\mathrm{CH}_{4}$ & -.526 & $.630^{* *}$ & $.530^{*}$ & .418 & 1 & 1 & 1 & 1 \\
\hline $\mathrm{W}_{\mathrm{S}}$ & -.048 & -.066 & -.063 & $.624^{*}$ & -.329 & -.289 & .287 & -.276 \\
\hline DIC & .720 & .005 & $.818^{* *}$ & $.694^{* *}$ & -.724 & $-.567^{*}$ & .387 & .291 \\
\hline $\mathrm{NH}_{4}^{+}$ & .453 & .139 & $.783^{* *}$ & -.255 & -.536 & -.183 & .409 & -.142 \\
\hline $\mathrm{NO}_{3}{ }^{-}+\mathrm{NO}_{2}{ }^{-}$ & -.314 & -.003 & .345 & $.689^{* * *}$ & $.884^{* * *}$ & -.120 & $.661^{* * *}$ & .158 \\
\hline TDN & .285 & $-.576^{*}$ & $.692^{* *}$ & .351 & -.341 & $-.527^{*}$ & $.579 * *$ & .335 \\
\hline DON & .278 & -.467 & $.600^{* *}$ & 297 & -.333 & -.235 & $.554^{* *}$ & .236 \\
\hline PN & -.071 & -.093 & .387 & .475 & -.001 & -.346 & -.192 & .403 \\
\hline ТP & .597 & -.132 & .033 & .346 & -.211 & .175 & -.117 & -.118 \\
\hline TDP & $.794^{*}$ & .014 & $.539^{* *}$ & $.616^{*}$ & -.285 & .251 & .397 & -.025 \\
\hline $\mathrm{PC}$ & .669 & -.141 & .359 & $.526^{*}$ & -.743 & -.401 & -.146 & $.613^{*}$ \\
\hline DOC & $.913^{* *}$ & $-.670^{* *}$ & $.462^{*}$ & -.510 & -.622 & $-.552^{*}$ & .396 & -.034 \\
\hline $\mathrm{Cl}^{-}$ & .227 & .044 & $.458^{*}$ & $.641^{*}$ & -.680 & -.303 & .329 & .149 \\
\hline $\mathrm{SO}_{4}{ }^{2-}$ & .154 & $-.582^{*}$ & -.291 & $.668^{* *}$ & .004 & $-.706^{* *}$ & -.027 & .282 \\
\hline $\mathrm{Na}^{+}$ & -.315 & $-.481^{*}$ & $.654^{* *}$ & $.715^{* *}$ & -.376 & $-.573^{*}$ & $.474^{*}$ & .331 \\
\hline $\mathrm{K}^{+}$ & .156 & .147 & $.793^{* *}$ & $.712^{* *}$ & -.644 & -.215 & $.467^{*}$ & .416 \\
\hline $\mathrm{Ca}^{2+}$ & .561 & -.340 & $.587^{* *}$ & $.703^{* *}$ & -.014 & $-.824^{* *}$ & .282 & .200 \\
\hline $\mathrm{Mg}^{2+}$ & .349 & -.364 & .327 & $.718^{* * *}$ & -.478 & $-.751^{* *}$ & $.448^{*}$ & .380 \\
\hline $\mathrm{Fe}$ & $.974^{* *}$ & $-.575^{*}$ & $.741^{* *}$ & -- & -.483 & -.029 & .278 & -- \\
\hline Alkalinity & .746 & -.015 & $.818^{* * *}$ & $.697^{* *}$ & $-.755^{*}$ & $-.592^{* * *}$ & $.487^{*}$ & .266 \\
\hline $\mathrm{HCO}_{3}^{-}$ & $.783^{*}$ & -.015 & $.818^{* *}$ & $.698^{* *}$ & -.744 & $-.591^{* *}$ & $.487^{*}$ & .266 \\
\hline $\mathrm{CO}_{3}{ }^{2-}$ & -.472 & -- & -- & -- & -.287 & -- & -- & -- \\
\hline TDS & .436 & -.150 & $.439^{*}$ & -.230 & -.251 & -.383 & .360 & .011 \\
\hline Chl-a & -.636 & -.317 & .277 & -.068 & .131 & -.251 & -.076 & -.039 \\
\hline $\mathrm{pH}$ & $-.854^{*}$ & -.392 & -.169 & -.261 & .088 & $-.512^{*}$ & -.073 & -.175 \\
\hline
\end{tabular}

Evap.: Evaporative ponds; Melt.: Meltwater systems; Shore.: Shoreline ponds; LH Shore: Lake Hazen Shoreline; Air ${ }_{\mathrm{P}}$ : barometric pressure; Water $\mathrm{T}_{\mathrm{T}}$ : water temperature; $\mathrm{dCO}_{2}$ : dissolved carbon dioxide concentration; $\mathrm{dCH}_{4}$ : dissolved methane concentration; $\mathrm{W}_{\mathrm{S}}$ : wind speed; DIC: dissolved inorganic carbon; $\mathrm{NH}_{4}{ }^{+}$: ammonium; $\mathrm{NO}_{3}{ }^{-} \mathrm{NO}_{2}{ }^{-}$: nitrate + nitrite; TDN: total dissolved nitrogen; DON: dissolved organic nitrogen; PN: particulate nitrogen, TP: total phosphorus; TDP: total dissolved phosphorus; PC: particulate carbon; DOC: dissolved organic carbon; $\mathrm{Cl}^{-}$: chloride; $\mathrm{SO}_{4}{ }^{2-}$ : sulphate; $\mathrm{Na}^{+}$: sodium; $\mathrm{K}^{+}$: potassium; $\mathrm{Ca}^{2+}$ : calcium; $\mathrm{Mg}^{2+}$ : magnesium; Fe: total iron; $\mathrm{HCO}_{3}{ }^{2-}$ : bicarbonate; $\mathrm{CO}_{3}{ }^{2-}$ : carbonate; TDS: total dissolved solids; Chl-a: chlorophyll-a; $\mathrm{pH}: \log \left[\mathrm{H}^{+}\right]$ 
Table S5 Freshwater ebullition fluxes of carbon dioxide $\left(\mathrm{CO}_{2}\right)$ and methane $\left(\mathrm{CH}_{4}\right)$ during the growing seasons of 2007 and 2008 in Skeleton Lake and Pond 01 in the Lake Hazen watershed.

\begin{tabular}{cccccc} 
Pond/Lake & $\begin{array}{c}\text { Bubble } \\
\text { volume } \\
(\mathbf{m L})\end{array}$ & $\begin{array}{c}\mathbf{C O}_{\mathbf{2}} \text { Bubble } \\
\text { concentration } \\
\left(\mathbf{m g C O}_{\mathbf{2}} \mathbf{L}^{-\mathbf{1}}\right)\end{array}$ & $\begin{array}{c}\mathbf{C O}_{\mathbf{2}} \mathbf{B u b b l e} \\
\mathbf{f l u x} \\
\left(\mathbf{m g C O}_{\mathbf{2}} \mathbf{~ m}^{-\mathbf{2}} \mathbf{d}^{-\mathbf{1}}\right)\end{array}$ & $\begin{array}{c}\mathbf{C H}_{\mathbf{4}} \mathbf{B u b b l e} \\
\mathbf{c o n c e n t r a t i o n} \\
\left(\mathbf{m g C H}_{\mathbf{4}} \mathbf{L}^{-\mathbf{1}}\right)\end{array}$ & $\begin{array}{c}\mathbf{C H}_{\mathbf{4}} \mathbf{B u b b l e} \\
\text { flux } \\
\left(\mathbf{m g C H}_{\mathbf{4}} \mathbf{~ m}^{-\mathbf{2}} \mathbf{d}^{-\mathbf{1}}\right)\end{array}$ \\
\hline Pond 01 & & & & & \\
30-Jun.-2007 & 1.76 & 0.60 & $0.06 \pm 0.01$ & 0.07 & $0.01 \pm 0.00$ \\
10-Jul.-2007 & 2.01 & 0.33 & $0.00 \pm 0.00$ & 0.09 & $0.00 \pm 0.00$ \\
17-Jul.-2007 & 1.07 & 18.53 & $0.13 \pm 0.01$ & 60.7 & $0.43 \pm 0.04$ \\
23-Jul.-2008 & 0.20 & 0.42 & $0.00 \pm 0.00$ & 0.18 & $0.00 \pm 0.00$ \\
31-Jul.-2008 & 1.17 & 0.53 & $0.04 \pm 0.03$ & 0.52 & $0.03 \pm 0.03$ \\
& & & & & \\
Skeleton L. & & & & & \\
04-Jul.-2007 & 5.51 & 0.46 & 0.01 & - & $-0.00 \pm 0.00$ \\
10-Jul.-2007 & 6.53 & 0.28 & $0.01 \pm 0.00$ & 0.00 & $0.00 \pm 0.00$ \\
17-Jul.-2007 & 5.86 & 0.29 & $0.01 \pm 0.00$ & 0.05 & $0.01 \pm 0.00$ \\
23-Jul.-2008 & 2.50 & 0.64 & $0.09 \pm 0.00$ & 0.04 & $0.00 \pm 0.00$ \\
31-Jul.-2008 & 2.00 & 0.61 & $0.07 \pm 0.06$ & 0.04 &
\end{tabular}


Squared Euclidean Distance

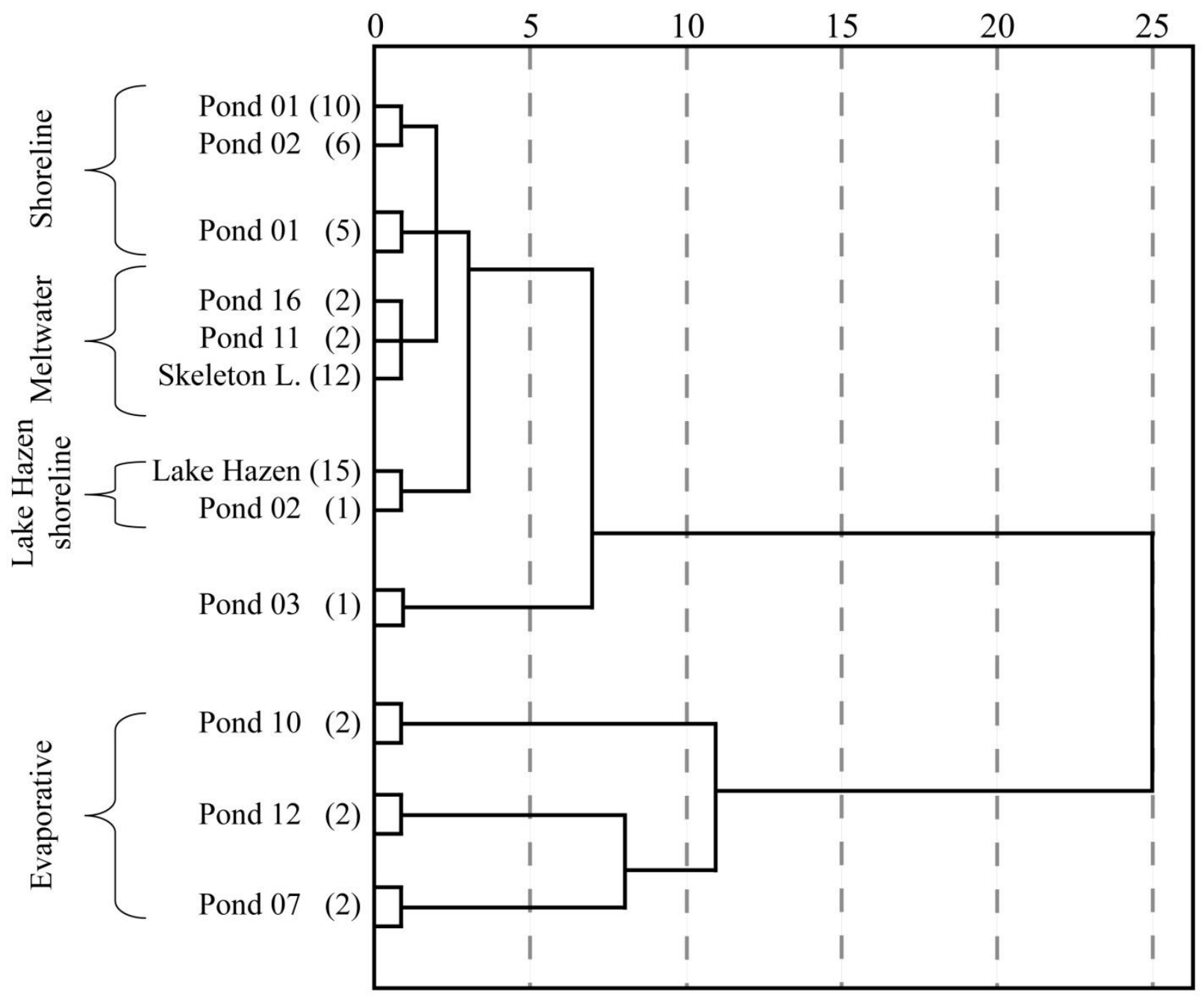

Figure S1 Dendrogram of sampled high Arctic freshwater systems in the Lake Hazen watershed during the growing season (June-August) between 2005, and 2007-2012 (hierarchical cluster analysis; see Methods). Water chemistry (see Methods) and carbon greenhouse gases concentrations measured periodically from 10 locations (Figure 1; Table 2, S2) were used as inputs to the analysis. Bracketed numbers represent the number of individual nodes (samples) that were compressed by site for ease of display. 


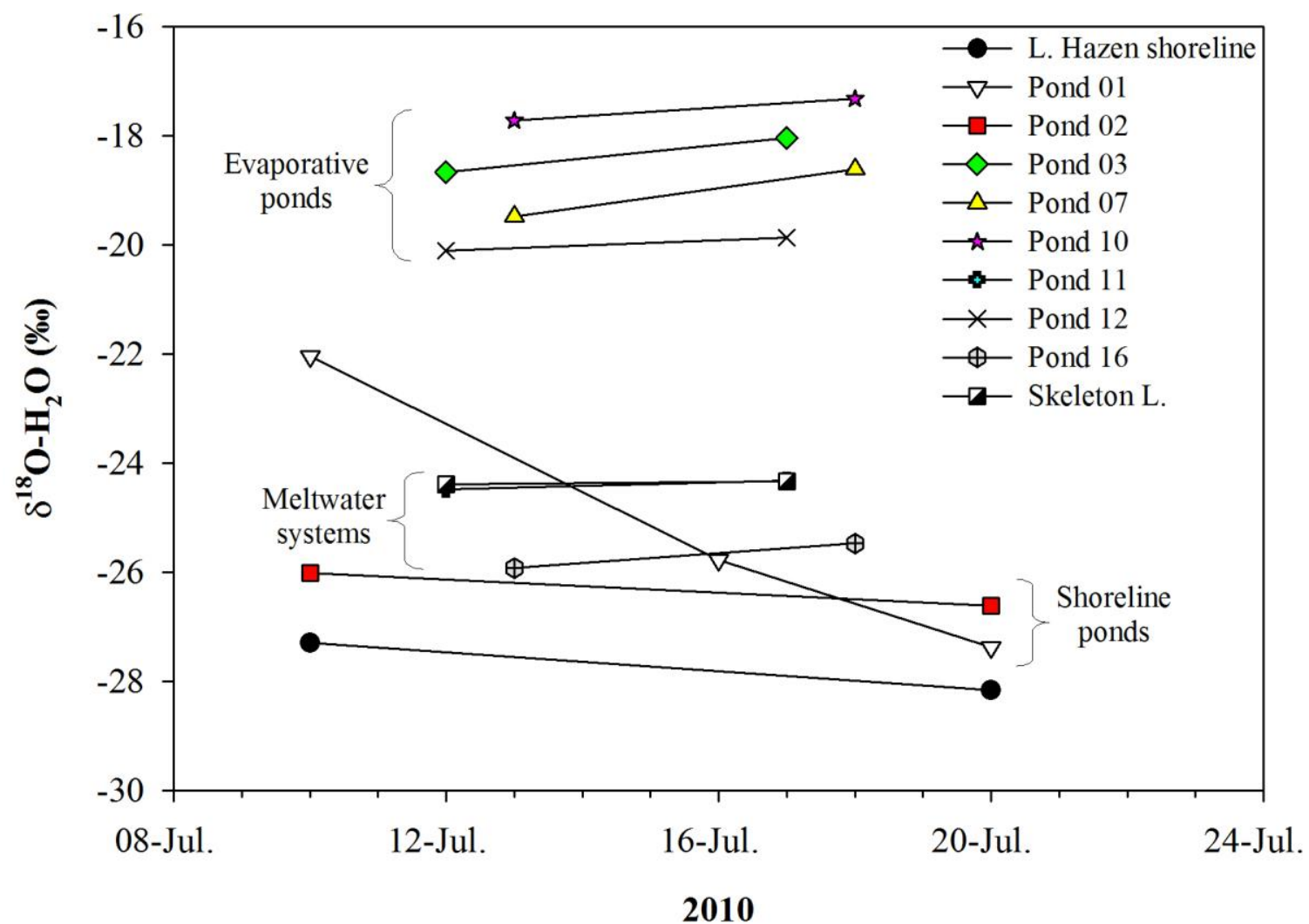

Figure S2 Measurements of stable oxygen isotopic composition of water $\left(\delta^{18} \mathrm{O}-\mathrm{H}_{2} \mathrm{O}\right)$ from ten freshwater systems in the Lake Hazen watershed in July 2010. Grouping of freshwater system types followed those delineated in the hierarchical cluster analyses (see Results and Discussion). 


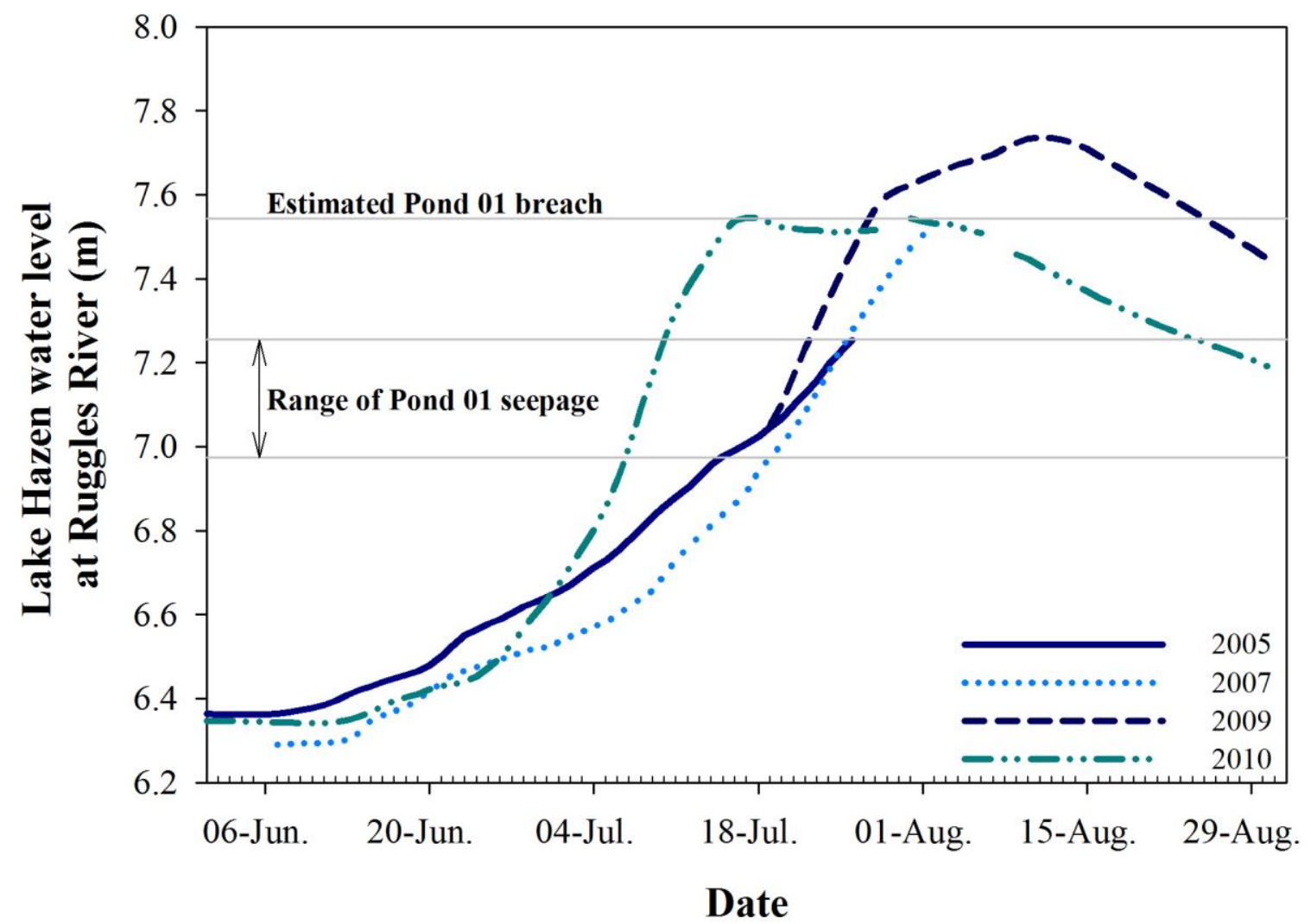

Figure S3 Lake Hazen water level data during the summer growing seasons of 2005, 2007, and 2009-10 at Ruggles River (Environment and Climate Change Canada, 2015). The range of water level when Pond 01 received Lake Hazen seepage water through its gravel berm is indicated and based on rapid changes in greenhouse gases concentrations. Rapid dilution of methane $\left(\mathrm{CH}_{4}\right)$ concentrations and field observations were used to determine the water level of pond breach and flushing. 

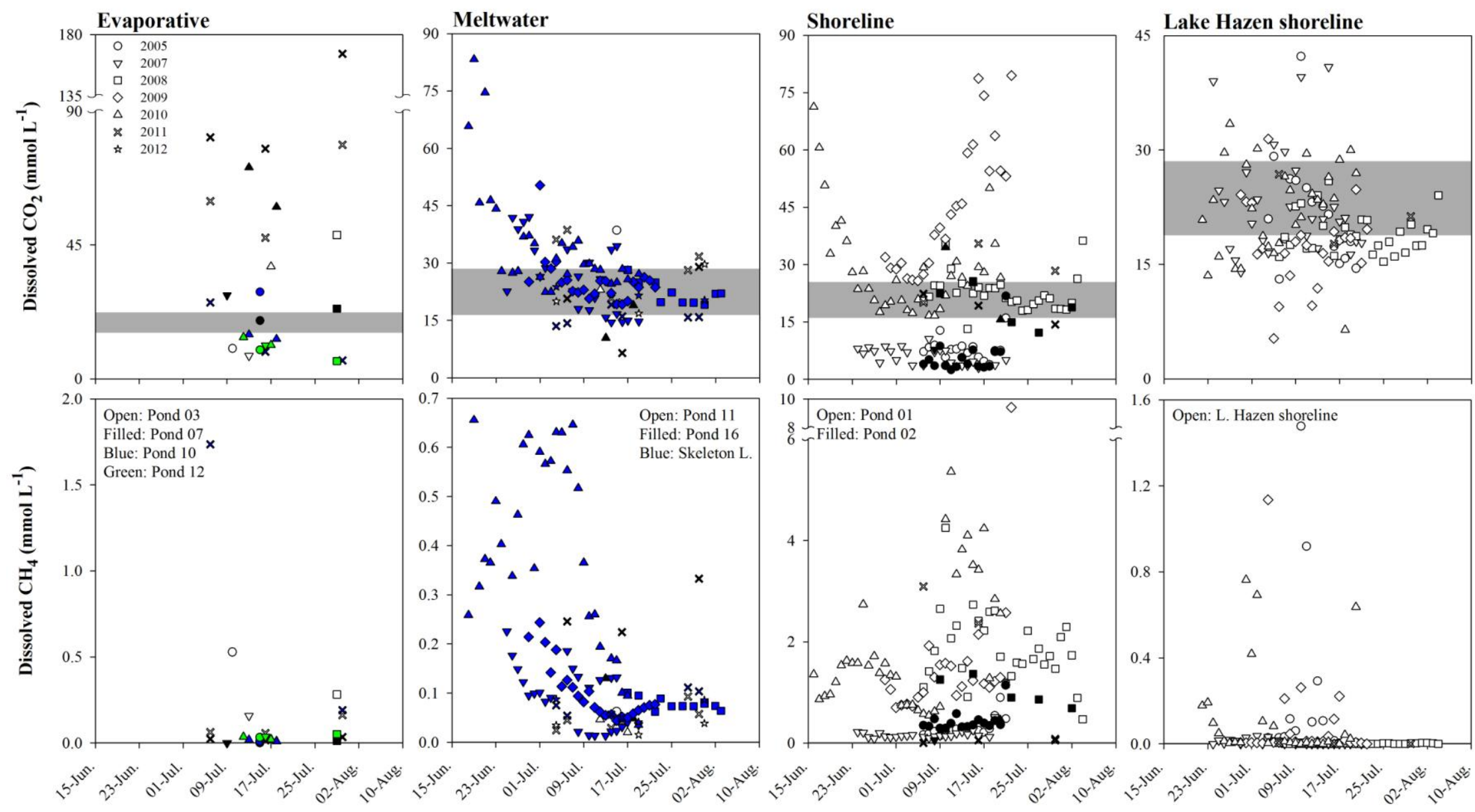

Figure S4 Individually-scaled dissolved carbon dioxide $\left(\mathrm{CO}_{2}\right)$ and methane $\left(\mathrm{CH}_{4}\right)$ concentrations during the 2005, and 2007-2012 growing seasons (June-August) from different types of high Arctic freshwater systems in the Lake Hazen watershed (also see Figure 2). Inset text shows site names within each freshwater type. Grey areas indicate the range of atmospheric equilibrium concentrations during the sampling period. 


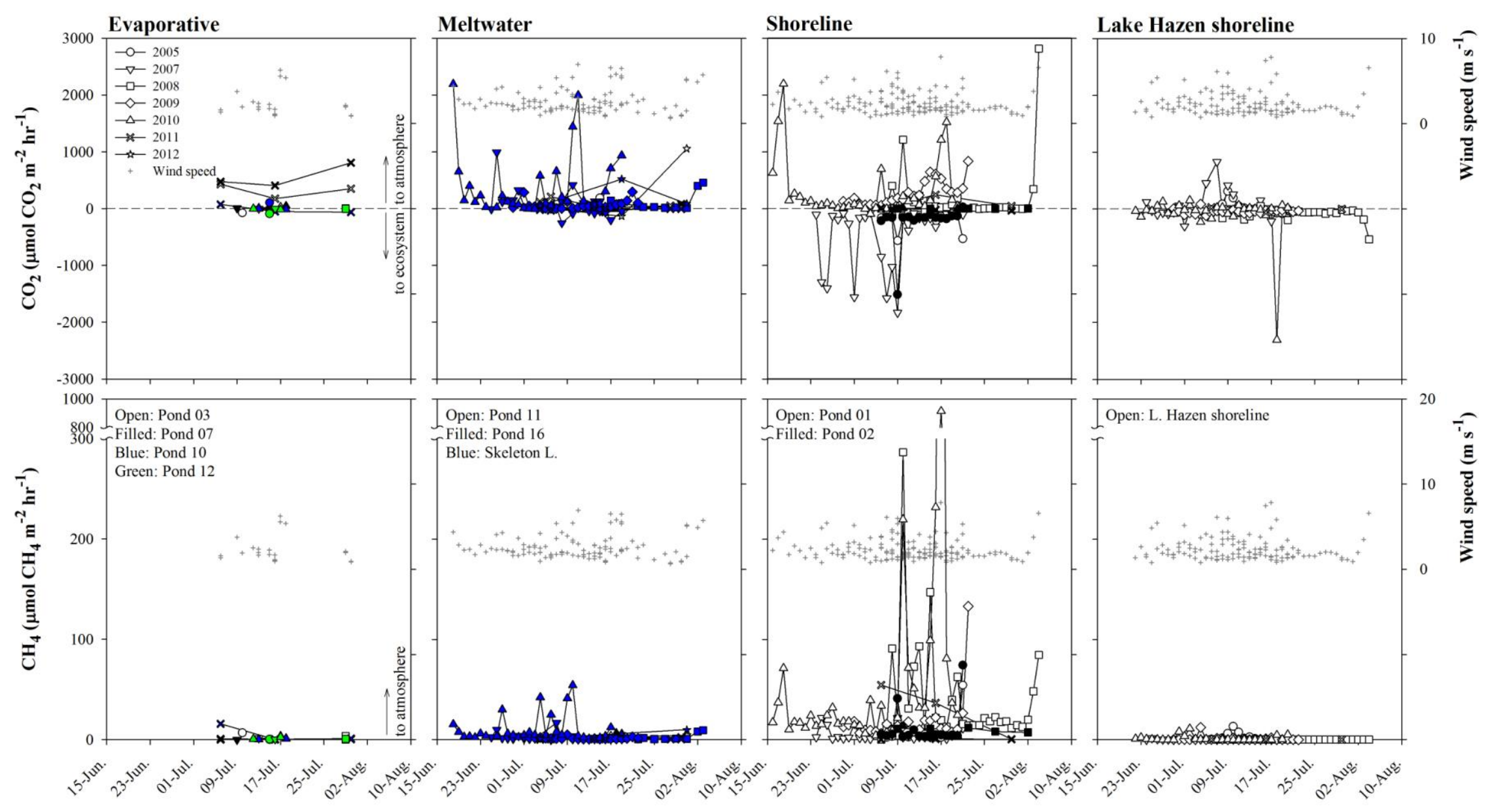

Figure S5 Carbon dioxide $\left(\mathrm{CO}_{2}\right)$ and methane $\left(\mathrm{CH}_{4}\right)$ fluxes during the 2005, 2007-2012 growing seasons (June-August) from four different freshwater types in the Lake Hazen watershed. Fluxes calculated using empirical equations and site conditions including water temperature, wind speed, barometric pressure and gas concentrations in water (see Methods). Daily wind speed from nearest measurements indicated by grey points. 


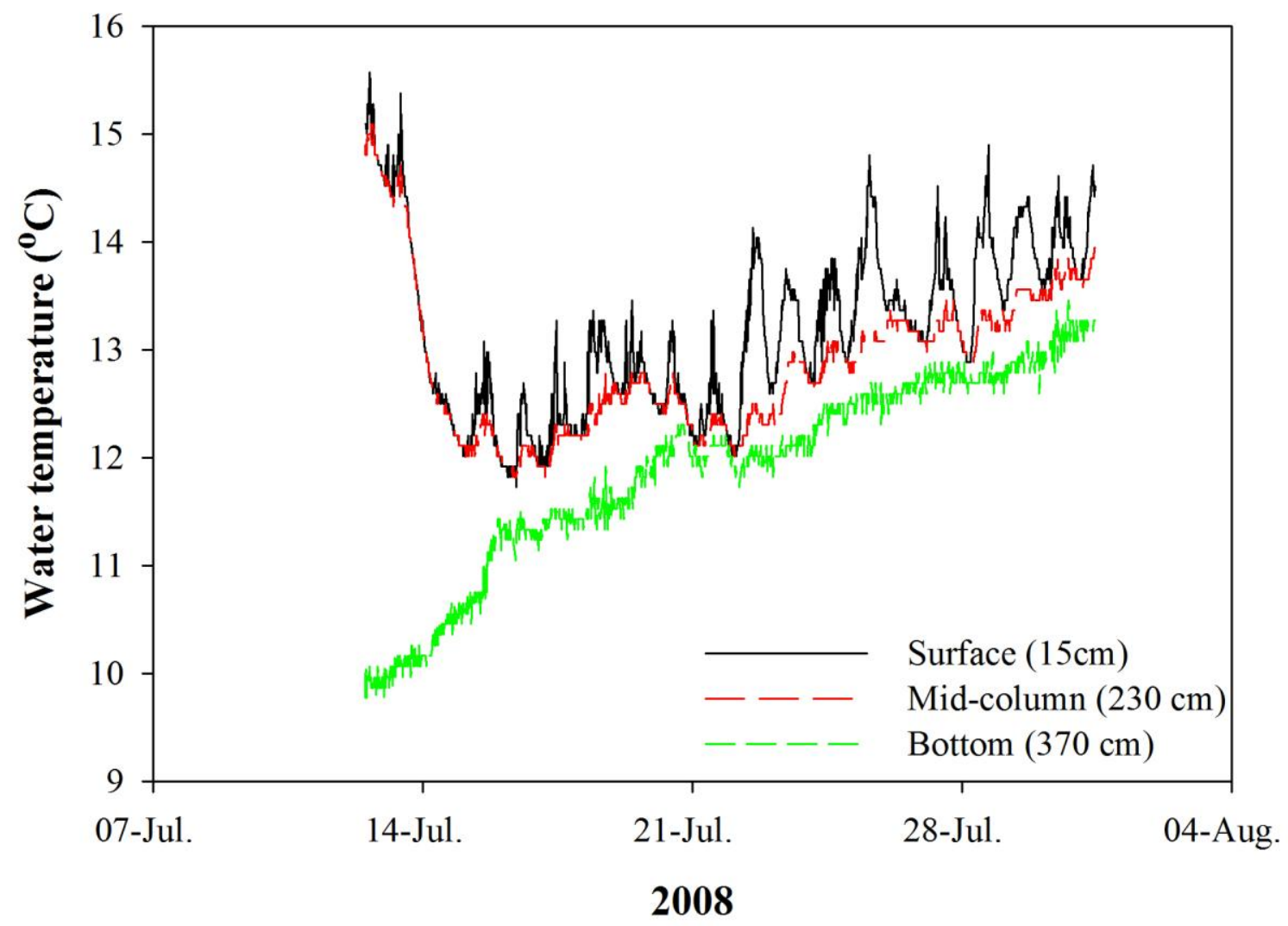

Figure S6 Water temperature stratification in Skeleton Lake (Meltwater pond) during the summer growing season of 2008 . 

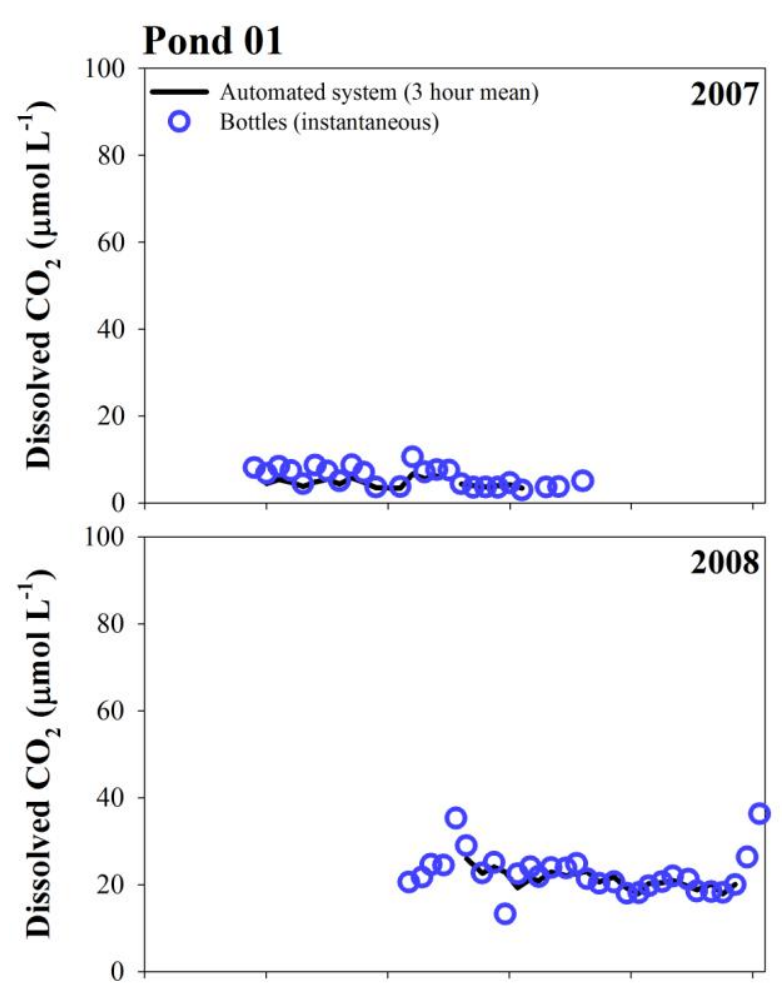

\section{Skeleton Lake}
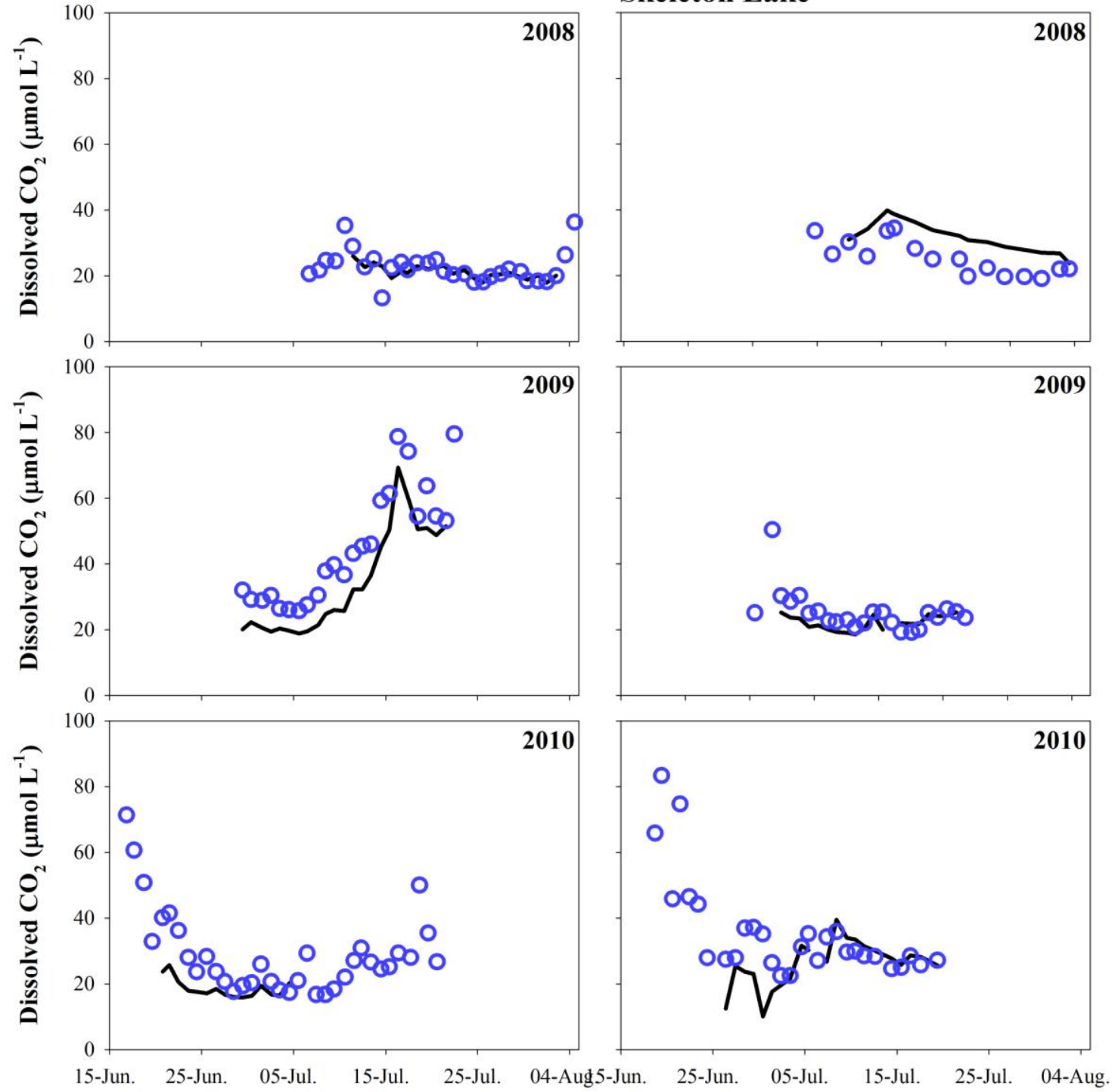

Figure S7 Comparison of dissolved $\mathrm{CO}_{2}$ concentrations between manually-collected bottle samples and automated systems in Pond 01 and Skeleton Lake during the growing seasons of 2007-2010. 


\section{References}

Hamilton JD, Kelly CA, Rudd JWM, Hesslein RH, Roulet NT (1994) Flux to the atmosphere of $\mathrm{CH}_{4}$ and $\mathrm{CO}_{2}$ from wetland ponds on the Hudson-Bay lowlands (hbls). Journal of Geophysical Research-Atmospheres, 99, 1495-1510.

Environment and Climate Change Canada, Water Survey of Canada. (2015) Real time hydrometric data. Available from: https://www.ec.gc.ca/rhc-wsc/. 